\title{
Quotient Evolutionary Space: Abstraction of Evolutionary process w.r.t macroscopic properties
}

\author{
Amhedkar Dukkipati, M. Narasimha Murty and Shalahh Bhatnagar \\ Department of Computer Science and Automation \\ Indian Institute of Science \\ Bangalore-560012, India \\ \{ambedkar,mnm, shalabh\}@csa 1 sc ernet in
}

\begin{abstract}
Darwinian evolution, which is characterized in terms of particular macroscopic behavior that emerges from microscopic organismic interaction, considers populations as units of evolutionary change. We formalize these concepts in evolutionary computationby developing notion of quotient evolutionary space(Q.E.S). We map set of all finite populations to a set of macroscopic properties of population those are chosen $a$ prior;; and we call this mapping as evolutionary criteria. On the 'quotient set of populations' that is induced by evolutionary criteria, we define mathematicalstructures to define evolutionary change with respect to chosen macroscopic parameters at populational level. This allows us to transform the objective defined on the search space that is imposed by the fitness function to an objective on the population space. We call quotient set of populations along with the mathematical structures the quotient evolutionary space. Tn demonstrate the ab. straction we consider fitness distribution of population as evolutionary criteria and give a detailed analysis of resulting spaces and basic convergence results.
\end{abstract}

\section{Introduction}

Most theories of evolutionary algorithms stress on particular aspects of evolutionary computation by means of specific mechanisms. Evolutionary algorithms can he modeled as Markov chains in a very natural way. For detailed analysis of evolutionary algorithms using Markov chains one can refer to $[\mathrm{I}, 2,3]$. This approach can be applied to evolutionary algorithms with finite population based on any kind of selection mechanism and evolution operators. The most difficult issue, however, is that it is impossible or at least impractical to formulate the details of the related transition probability matrix and, therefore, analysis of the properties of the matrix is difficult [4].

Statistical mechanics based theory of genetic algorithms makes use of fitness distributions and analyzes change in the distrihution of fitnesses in the population from one generation to the next [5]. Changes in the fitness distrihutions are studied through the average evolution of the first few cumulants of the fitness distrihutions. For the detailed theory, one can refer to $[6,7,8]$. Denoting the distribution of fitnesses at generation $t$ by $\rho^{t}$, the approach is based on the schematic representation:

$$
\rho^{t} \stackrel{\text { selection }}{\longrightarrow} \rho_{s}^{t} \stackrel{\text { mutätion }}{\longrightarrow} \rho_{s m}^{t} \stackrel{\text { crossover }}{\longrightarrow} \rho_{s m c}^{t}=\rho^{t+1}
$$

This approach is developed for genetic algorithms and can be extended to other paradigms of evolutionary computation, however, it considers only specific selection mechanisms (for example, Boltzmann selection). This approach depends on the particular fitness functions (fitness is a function of the magnetization or Hamming distance to all-ones optimum [9]).

In general, theoretical models of evolutionary computation formalize evolutionary process as a process composed of selection and evolutionary operators. This approach assigns the 'mechanism' status to natural selection rather than one of a generalized process which brings in 'evolutionary change'. But the modern evolutionary perspective views "natural selection" as an abstract process which brings in evolutionary change rather as a particular mechanism by which evolution is carried out. These aspects are important to develop a 'unified' theory of evolutionary computation.

Fitness landscape, the concept which was introduced in evolutionary biology to view evolutionary process as a hill-climbing-like process (one can consider fitness function as a fitness landscape in the framework of evolutionary algorithms) is of growing interest today in the fields of evolutionary computation and theoretical models of molecular evolution. There have been theoretical frameworks to characterize fitness landscapes. These frameworks impose a 'neighborhood' relation on individuals with respect to particular evolutionary operators like selection, mutation etc. [10]. In general, this approach introduces some sort of additional geometric, topological or algebraic structure on the search space that allows one to define closedness. similarity of individuals in the search space [11]. The main drawbacks of the above approach to comprehend the evolutionary process are the following:

- At any given time, elements of a population are distributed all over the fitness landscape and it is very difficult to get the population dynamics as a whole.

- The concept of fitness landscape should he independent of any particular evolutionary mechanism. (For example, in evolutionary algorithms, fitness function 
as fitness landscape is independent of the evolutionary mechanism. Fitness landscape is considered independent of mechanism in some molecular evolutionary models also [12]).

- Evolutionary process is a populational process [1.3]. Hence evolutionary change should be defined at the population level rather than at individual level.

In this paper we develop a mathematical abstraction of evolutionary computation, where we give 'strict' status of populational process for evolutionary algorithms by posing mathematical structure on the space of populations rather than on the search space. We deal with the populations by mapping these to their macroscopic properties, which we call 'criterion set' and we call this map as 'evolutionary criteria'. Based on this concept, we develop notion of 'quotient evolutionary space' (Q.E.S) with respect to which we abstract evolutionary process. The features of abstraction that we are going to present in this paper are:

- Main difference between evolutionary algorithms and stochastic search methods is that the former maintain a population of candidate solutions, instead of one candidate solution at any given point of time. Hence we abstract evolutionary process as a populational process.

- Abstraction is independent of any particular macroscopic structure of population; it is based on the concept of evolutionary criteria which can be instantiated with any specific macroscopic properties of population.

- Mathematical structures are posed on the set of all populations so that the objective (due to fitness function) defined on the search space is transformed to an objective on the population space and to measure the evolutionary changes with respect to the chosen macroscopic properties.

- Many theories of evolutionary algorithms deal with infinite populations. Since computational complexity of these algorithms is defined in terms of number of fitness evaluations, we assume that population size is finite.

- Evolutionary process is defined as an abstract process which brings in evolutionary changes instead of defining it in terms of particular evolutionary mechanism.

The outline of paper is as follows. In $\S 2$, we give basic definitions and some assumptions for the framework that we are going to develop. In $\S 3$ we formalize the concept of evolutionary criteria. We give a formal definition of quotient evolutionary space and abstract notion of evolutionary process in $\S 4$. Finally in $\S 5$, we give a detailed analysis of resulting spaces and evolutionary process when evolution- criteria is fitness distribution

\section{Towards Abstraction}

\subsection{System under consideration}

Evolutionary computation uses the metaphor of mapping "problem solving" onto a simple model of evolution as shown below [14].

\begin{tabular}{ccc}
\hline \hline Evolution & $\Longleftrightarrow$ & Problem Solving \\
\hline environment & $\longleftrightarrow$ & problem \\
organism & $\longrightarrow$ & candidate solution \\
fitness & $\longleftrightarrow$ & quality \\
\hline
\end{tabular}

Set of all candidate solutions is called the search which is denoted by $\Omega$. The problems which we intend to solve by evolutionary computation technique offer (or one should design) a fitness function (or objective function) $f$ : $\Omega \rightarrow \mathbb{R}$. The goal of an evolutionary algorithm is:

given a search space $\Omega$, objective function $f$ :

$\Omega \rightarrow \mathbb{R}$, find $\omega_{0} \in \Omega$ such that,

$$
f(\omega) \leq f\left(\omega_{0}\right) \quad \forall \omega \in \Omega
$$

$\mathbf{P}$ is a set of all non empty multi-subsets of $\Omega$. $\mathbf{P}$ represents the set of all possible populations and $P \in \mathcal{P}$ is a particular population. $n_{P} \in \mathbb{Z}^{+}$denotes the population size and is assumed to be finite.

\subsection{Basic Definitions}

The most important mechanism for evolutionary algorithms is selection. Selection mechanisms depend only on the fitness distribution of the population [6]. In this paper, we consider fitness distribution as an example of instantiation of evolutionary criteria (i.e., macroscopic property of population).

One important property of fitness distribution of a finite population is that only for finitely many points its value is non-zero, since population size is assumed to be finite. Here we give a generalized definition of fitness distribution which is independent of population.

Definition 2.1 'Fitness distribution' is afunction $\rho: \mathbb{R} \rightarrow$ $Z^{+} \cup\{0\}$ which is bounded and satisfies

$$
\sharp\{x: \rho(x) \neq 0\}<\infty,
$$

where $\sharp$ denotes the cardinality of a set.

Definition 2.2 'Fimess distribution of a population' $P=$ $\left\{\omega_{k}\right\}_{k=1}^{n_{P}}$ is afunction $\rho^{P}: \boldsymbol{R} \rightarrow \mathbb{Z}^{+} \cup\{0\}$ defined as

$$
\rho^{P}(x)=\sum \delta\left(x-f\left(\omega_{k}\right)\right)
$$

where $6: \boldsymbol{R} \rightarrow\{0.1)$ is the Kronecker delta function de. fined as $\delta(x)=1$ if $x=0, \delta(x)=0$ otherwise. 
$\rho^{P}$ assigns each $x \in \boldsymbol{R}$ to the number of individuals in a population $P$ carrying $x$ as the fitness value. Since we assume that population size is finite, fitness distribution of a population is indeed a fitness distribution. The finite set of values associated with the fitness distribution which are mapped to non-zero values is called support of fitness distribution of population.

Definition 2.3 Let $\rho^{P}$ be the fitness distribution of population $P_{t}$ then 'support' of $\rho^{P}$ is defined as

$$
\operatorname{support}\left(\rho^{P}\right)=E_{P}=\left\{x: \rho^{P}(x) \neq 0\right\},
$$

which is a finite set.

Size of population can be expressed in terms of fitness distribution as follows:

The most important statistical properties of the population are the mean $\mu$, and the variance $\sigma^{2}$ of fitness distribution of population [7].

Definition 2.4 Mean of fitness distribution of a population $P \in P$ with fitness distribution $\rho^{P}$ is defined as

$$
\mu\left(\rho^{P}\right)=\frac{1}{--} \quad x \rho^{P}(x) .
$$

Definition 2.5 Variance offitness distribution of a population $P \in P$ with fitness distribution $\rho^{P}$. is defined as

$$
\sigma^{2}\left(\rho^{P}\right)=\perp \quad\left(x-\mu\left(\rho^{P}\right)\right)^{2} \rho^{P}(x) .
$$

\subsection{The Approach}

Darwinian evolution, which substituted typological thinking with populational thinking, considers population as the basic unit of evolutionary change. One naive approach to formalize evolutionary process in evolutionary algorithms is to consider each ingredient of population. For example, the state of a genetic algorithm can he described as a vector on the simplex of a high-dimensional Euclidean space [15]. The genetic algorithm dynamics is specified by a nonlinear matrix operator that acts on this vector to produce the slate at the next time step. Although this formalism exactly captures the detailed 'microscopic' dynamics of the algorithm, in practice the large size of these matrices makes it impossible to obtain quantitative results [16]. Also the difficulty is, however, that the precise expression for the related nonlinear mapping can be obtained only for very few cases (e.g., a binary GA with proportional selection).

Modeling of evolutionary algorithms based on the macroscopic properties of population is not a new concept and has been given in [5], based on the specific macroscopic parameters like 'cumulants of fitness distributions'. In this paper, instead of choosing specific macroscopic parameters of population, we introduce a concept called 'evolutionary criteria' which comprises all macroscopic properties of population that one can choose. Note that the macroscopic properties or criteria we choose should be able to characterize the population, by means of which, in an ideal case, we should be able to predict and control the evolutionary process. For example, for an evolutionary algorithm for optimization problems, one can choose maximum fitness of population as a criterion. Though one would be interested in observing maximum fitness, it is not enough to capture the evolutionary process.

Formally we define evolutionan criteria as a function defined from set of all populations to criteria set. Criteria set is a set of all macroscopic properties one would choose. We establish a bijection from the quotient set (set of all classes of populations with the same criteria) that is given by evolutionary criteria to a subset of criteria set. Hence we pose a mathematical structure on criteria set which induces the same on the quotient set of populations. Two important mathematical structures that we pose on criterion set are evolutionary order and evolutionary metric. Evolutionary order is a partial order that is defined on criteria set to define a objective on the population space based on the objective that we already have on the search space. Evolutionary metric is a metric defined on the criteria set to measure the evolutionary change. Quotient set of populations along with evolutionary order and evolutionary metric is called quotient evolutionary space (Q.E.S).

After formalizing above concepts we define evolutionary process with respect to chosen criteria, implicitly as an optimization process.

\section{Evolutionary Criteria: From search space to population space}

\subsection{Formalization of Evolutionary Criteria}

Let $\mathcal{Q}$ be the criteria set which represents the macroscopic properties of population, that are chosen apriori. Since one can choose more than one macroscopic property, $\mathcal{Q}$ can be written as $\mathcal{Q}=\left(\mathcal{Q}_{1}, \mathcal{Q}_{2}, \ldots \mathcal{Q}_{l}\right)$ : where $\mathcal{Q}_{i}$ represents a macroscopic property $\forall i=1 \ldots 1$. We define evolutionary criteria as follows.

Definition 3.1 Evolutionary criteria is a function $3: \mathcal{P} \rightarrow$ $\mathcal{Q}$, where $\mathcal{P}$ is set of all populations and $\mathcal{Q}$ is a criteria set. Note that choice of evolutionary criteria $\mathcal{F}$ depends on the fitness function $f: \Omega \rightarrow \boldsymbol{R}$ since the important macroscopic properties of a population depends on the fitness values of individuals (see Figure 1).

Function 3 partitions the set of all populations $P$ into equivalence classes according to kernel equivalence $\sim_{\mathcal{F} \subseteq}$ 


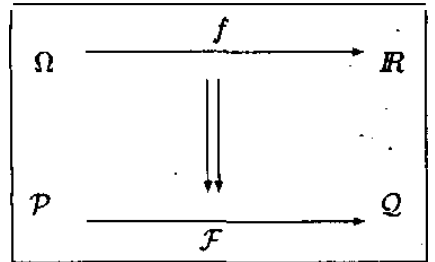

Figure 1: Evolutionary criteria: from search space to population space

$P \times P$ defined as,

$$
P_{1 \sim \mathcal{F}} P_{2} \Longleftrightarrow \mathcal{F}\left(P_{1}\right)=\mathcal{F}\left(P_{2}\right) \quad \forall P_{1}, P_{2} \in P
$$

Respective quotient set (set of all equivalence classes) $\mathcal{P} / \sim \mathcal{F}$ is defined as,

$$
\mathcal{P} / \sim \mathcal{F}=\{[P]: P \in \mathcal{P}\}
$$

where $[P] \mathbf{c} P$ is an equivalence class i.e.,

$$
[P]=\left\{P^{\prime}: P^{‘} \sim_{\mathcal{F}} P\right\} \forall P \in P
$$

We call $\mathcal{P} / \sim_{\mathcal{F}}$ as, quotient set of populations. w.r.t evolutionary criteria 3 . The quotient map $\pi_{\mathcal{F}}: P \rightarrow \mathcal{P} / \sim_{\mathcal{F}}$ is defined as

$$
\pi_{\mathcal{F}}(P)=[P] \cdot \forall P \in \mathcal{P}
$$

Quotient map is nothing but a function which maps each element to its corresponding equivalence class. Observe that $\pi_{\mathcal{F}}$ is an "on-to" function.

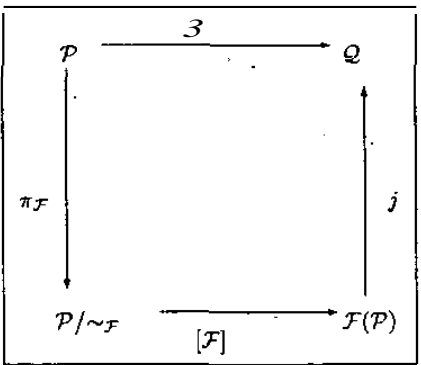

Figure 2: Getting the, bijective mapping from quotient population space $\mathcal{P} / \sim_{\mathcal{F}}$ to subset of criteria set $\mathcal{F}(\mathcal{P}) \mathrm{C} \mathcal{Q}$

But we would need, a bijective function which maps $\mathcal{P} / \sim_{\mathcal{F}}$ to $\mathcal{F}(\mathcal{P})$ to assign unique criteria value to each class of populations. Existence and uniqueness of this function is confirmed by the following theorem:

Theorem 3.2 Let $\mathcal{F}: P \rightarrow \dot{\mathcal{Q}}$ be afunction and $\sim_{\mathcal{F}}$ be the kernel equivalence of the map $\mathcal{F}$. Let $\mathcal{P} / \sim \mathcal{F}$ be the corresponding quotient set and $\pi_{\mathcal{F}}$ be the quotient map. Let $j: \mathcal{F}(\mathcal{P}) \rightarrow \mathcal{Q}$ be an “in-to"function defined as $j(Q)=$
$Q . \mathrm{VQ} \in \mathcal{F}(\mathcal{P})$ (which is termed as canonical injection). Then there is a unique map $[\mathcal{F}]: \mathcal{P} / \sim_{\mathcal{F}} \rightarrow \mathcal{F}(\mathcal{P})$ such that

$$
\mathcal{F}=j o[\mathcal{F}] \circ \pi \mathcal{F}
$$

Further $[\mathcal{F}]$ is bijective.

(For the proof of Theorem 3.2 see Appendix $\S$ A)

Figure 2 demonstrates all the mappings involved in getting the bijection $[\mathcal{F}]$, which allows one to identify each class of populations with an element from the criteria set. Note that $[\mathcal{F}]$ only maps $\mathcal{P} / \sim_{\mathcal{F}}$ to subset of criteria set $\mathcal{Q}$ namely $\mathcal{F}(\mathcal{P})$. Also the definition of function $[\mathcal{F}]$ : $\mathcal{P} / \sim \mathcal{F} \rightarrow \mathcal{F}(\mathcal{P})$ can be deduced as (see Appendix $\S \mathrm{A}$ ):

$$
[\mathcal{F}]([P])=\mathcal{F}(P) \quad \forall[P] \in \mathcal{P} / \sim \mathcal{F} .
$$

A trivial example of choosing $\mathcal{Q}$ is to set $\mathcal{Q}=\mathbb{R}$ and assign average fitness or maximum fitness to each population as an evolutionary criteria.

Note that evolutionary criteria can have more than one macroscopic property of population. In this paper we have considered only one macroscopic property, i.e., fitness distribution and have given the analysis for it.

Since the aim of this abstraction is to capture evolutionary change at populational level in terms of macroscopic parameters, we pose two basic mathematical structures on the quotient set of populations viz., evolutionary order and evolutionary metric.

\subsection{Evolutionary Order}

Not every change in the system can be termed as an evolutionary change [17]. One candefine evolutionary change as "sustainable change over a succession of generations" [18]. In this paper we consider, 'sustainable change' as a directional change towards a particular goal. Evolutionary process is a populational process hence, one has to define sustainable change at the level of populations. Since from the evolutionary algorithms perspective 'objective' is defined on the search space imposed by a fitness function, we have to transform the objective on the search space to an objective on the population space. For this purpose we pose mathematical order on the quotient' set of populations which induces the same on $\mathcal{P} /-3$ by the function $[\mathcal{F}]: \mathcal{P} / \sim_{\mathcal{F}} \rightarrow \mathcal{F}(\mathcal{P})$, i.e., if $\preceq_{\mathcal{F}}$ is partial order defined on $\mathcal{Q}$ then, $\forall\left[P_{1}\right],\left[P_{2}\right] \in \mathcal{P} / \sim_{\mathcal{F}}$

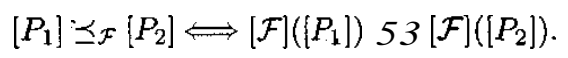

By (8) we define evolutionary order as follows.

Definition 3.3 A partial order $\preceq_{\mathcal{F}}$ defined on $\mathcal{Q}$, which is induced on $\mathcal{P} /-3$ by evolutionary criteria $3: P \rightarrow Q$ according to

$$
\left[P_{1}\right] \preceq_{\mathcal{F}}\left[P_{2}\right] \Longleftrightarrow \mathcal{F}\left(P_{1}\right) \preceq_{\mathcal{F}} \mathcal{F}\left(\dot{P}_{2}\right) \forall\left[P_{1}\right],\left[P_{2}\right] \in \mathcal{P} / \sim_{\mathcal{F}}
$$

is called evolutionary order. 
Note that the definition of $\preceq_{\mathcal{F}}$ should he compatible with the objective in the search space, for instance, maximization off : $\Omega \rightarrow \mathbb{R}$.

If we have $\mathcal{Q}=\mathrm{R}$ and $\mathcal{F}$ as assigning average fitness or maximum fitness of the population, the 'usual' order on $\mathbf{R}$ would define evolutionary order on $\mathcal{P} / \sim_{\mathcal{F}}$.

\subsection{Evolutionary Metric}

Evolutionary metric is the measure'of changes that take place in the system with respect to chosen criteria. To measure the evolutionary change with respect to chosen criteria 3 we define metric on' the set $\mathcal{Q}$ which induces same on $\mathcal{P} / \sim_{\mathcal{F}}$ by the function $[\mathcal{F}]: \mathcal{P} / \sim_{\mathcal{F}} \rightarrow \mathcal{F}(\mathcal{P})$, i.e., if $\boldsymbol{d}_{\mathcal{F}}$ is metric defined on $\mathcal{Q}$ then, $\forall\left[P_{1}\right],\left[P_{2}\right] \in \mathcal{P} / \sim_{\mathcal{F}}$

$$
d_{\mathcal{F}}\left(\left[P_{1}\right],\left[P_{2}\right]\right)=d_{\mathcal{F}}\left([\mathcal{F}]\left(\left[P_{1}\right]\right),[\mathcal{F}]\left(\left[P_{2}\right]\right)\right)
$$

From the Equation (8) we get

$$
d_{\mathcal{F}}\left(\left[\dot{P}_{1}\right],\left[P_{2}\right]\right)=d_{\mathcal{F}}\left(\mathcal{F}\left(P_{1}\right), \mathcal{F}\left(P_{2}\right)\right)
$$

Note that as the left side of $(9 \mathrm{a})$ and $(9 \mathrm{~b})$, the metric $d \mathfrak{F}$ is defined on $\mathcal{Q}$ while on the right side, the same is defined on $\mathcal{P} / \sim \mathcal{F}$. It should $\boldsymbol{h} \boldsymbol{e}$ clear from the context on which set the metric $d_{\mathcal{F}}$ is defined: Now the definition of evolutionary metric follows.

Definition 3.4 A metric $d_{\mathcal{F}}: Q x \mathcal{Q} \rightarrow \boldsymbol{R}$ dejined on $\mathcal{Q}$, which is induced on $\mathcal{P} / \sim_{\mathcal{F}}$ by evolutionary criteria $\mathcal{F}$ : $P \rightarrow \mathcal{Q}$ according to

$d_{\mathcal{F}}\left(\left[P_{1}\right]_{,}\left[P_{2}\right]\right)=d_{\mathcal{F}}\left(\mathcal{F}\left(P_{1}\right), \mathcal{F}\left(P_{2}\right)\right) \forall\left[P_{1}\right],\left[P_{2}\right] \in \mathcal{P} / \sim_{\mathcal{F}}$

is called evolutionary metric.

In $\S 3.1$, we defined criteria set $\mathcal{Q}$ as the tuple $\mathcal{Q}^{\cdot}=$ $\left(\mathcal{Q}_{1}, \mathcal{Q}_{2}, \ldots \mathcal{Q}_{l}\right)$ where $\mathcal{Q}_{i}$ is acriterion (macroscopic property) $\forall i=1 \ldots l$. But it is not easy to define a metric on $\mathcal{Q}$, because each $\mathcal{Q}_{i}$ represents a particular macroscopic property of population. Hence we can define metric $d_{\mathcal{F}}$ on $\mathcal{Q}$ as a product metric of metrics $d_{\mathcal{F}_{i}}$ defined on $\mathcal{Q}_{i}, \forall i=1 \ldots l$. One can choose metric $d_{\mathcal{F}}$ on $\mathcal{Q}$ as $d_{\mathcal{F}}=\max \left(d_{\mathcal{F}}\right)_{i=1}^{l}$ or $d_{\mathcal{F}}=\sum_{i=1}^{\ell} d \mathcal{F}_{i}$.

If the choice of evolutionary criteria is average fitness or maximum fitness of the population the metric would be 'usual' metric on $\mathbb{R}$.

\section{Evolutionary Process on Q.E.S}

\subsection{Q.E.S}

Based on the mathematical structures that we imposed on the quotient set of populations on the basis of the concept of evolutionary criteria, in this section we define evolutionary process, implicitly as an optimization process - the abstracfion we developed is sufficient enough to do so. Figure 3 gives the summary of the abstraction we developed. Formally we define Q.E.S as follows.

Definition 4.1 Q.E.S with respect to evolutionary criteria $3: P \rightarrow \mathcal{Q}$ is quotient set of populations $\mathcal{P} / \sim_{\mathcal{F}}$ induced by 3 , along with the evolutionay order 53 and evolutionary metric $d_{\mathcal{F}}$; and it is denoted as $\left(\mathcal{P} / \sim_{\mathcal{F}}, \$=d 3\right)$.

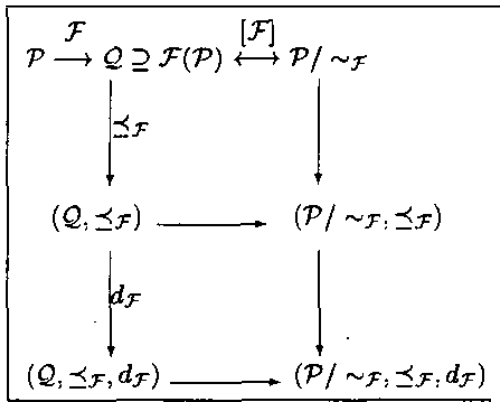

Figure 3: Population space to quotient evolutionary space

\subsection{Evolutionary Process}

A process in the evolutionary system can he written as as a sequence $\left\{\dot{P}_{n}\right\}$. where $P_{n} \in \mathcal{P}, V n=0,1,2 \ldots$ and $P_{0}$ is the initial population. But with respect to the evolutionary

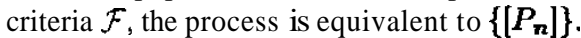

Definition 4.2 Given a sequence $\left\{P_{n}\right\} \subset \mathcal{P}, n=0,1 \ldots$ the equivalent process with respect to evolutionary criteria 3 is dejined as:

$$
\left\{\left[P_{n}\right]\right\} \subset \mathcal{P} / \sim \mathcal{F} \quad n=0,1, \ldots
$$

Since objective on search space is transformed to an ob. jective on quotient set of populations by evolutionary order, we define 'monotone evolutionary process' as follows.

Definition4.3 A sequence $\left\{P_{n}\right\} \subset \mathcal{P}, n=0,1 \ldots$ in the evolutionary system $\left(\mathcal{P} / \sim_{\mathcal{F}}, \preceq \mathcal{F}, d_{\mathcal{F}}\right)$ is said to be a mono. tone evolutionaryprocess if

$$
\left[P_{n}\right] \preceq \mathcal{F}\left[P_{n+1}\right] \quad \forall n
$$

Definition 4.4 An evolutionaryprocess $\left\{P_{n}\right\} \subset P$ is said to be convergent with respect to evolutionary criteria 3 if the sequence $\left\{\left[P_{n}\right]\right\}$ converges in $\mathcal{P} / \sim_{\mathcal{F}}$, i.e., $\exists P_{*} \in P \ni$

$$
\begin{gathered}
V e>0, \exists N=N(\epsilon) \in \mathcal{Z}^{+} 3 \\
n \geq N \Rightarrow d_{\mathcal{F}}\left(\left[P_{n}\right],\left[P_{*}\right]\right)<\epsilon .
\end{gathered}
$$

Remark 4.5 Evolutionary process $\left\{P_{n}\right\}$ converges with respect to evolutionary criteria iff $\left\{\mathcal{F}\left(P_{n}\right)\right\}$ converges in the space $\mathcal{Q}$. 


\section{Fitness Distribution as an Evolutionary Cri- teria}

$5.1\left(\mathcal{P} / \sim_{\rho}, \preceq_{\rho}, d_{\rho}\right)$

Let $\mathcal{Q}$ denote the set of all fitness distributions and $\mathcal{F}: \mathbf{P}$ $\mathcal{Q}$ be defined as $\mathcal{F}(P)=\rho^{P}, \forall P \in \mathrm{P}$ where $\rho^{P}$ is fitness distribution of $\mathbf{P}$. Also, quotient set with respect to this particular criteria is denoted by $\mathcal{P} / \sim_{\rho}$. To define QES with respect to chosen evolutionary criteria we have to specify evolutionary order $\preceq \rho$ and evolutionary metric $d$, on quotient set of populations.

Given that one is attempting to solve a maximization problem using evolutionary algorithms, one would expect that mean of the fitness distribution of population should shift towards right and one can also pose the condition that variance of population should decrease as the evolutionary process takes place. Hence we define partial order $\preceq_{\rho}$ as follows.

Definition 5.1 Lei $\mathcal{Q}$ represent the set of allfiiness distributions. Partial order $\preceq_{\rho} \subseteq \mathcal{Q} \times \mathcal{Q}$ is defined as $\forall \rho_{1}, \rho_{2} \in \mathcal{Q}$, $\rho_{1} \preceq_{\rho} \rho_{2}$ iff

$$
\mu\left(\rho_{1}\right) \leq \mu\left(\rho_{2}\right)
$$

and

$$
\sigma^{2}\left(\rho_{1}\right) \geq \sigma^{2}\left(\rho_{2}\right)
$$

where $\mu$ and $\sigma^{2}$ are the mean and variance offitness distribution respectively.

One can easily see that $\preceq_{\rho}$ is an partial order and hence it is an evolutionary order. Note that one can have different definitions, what we have given here is one of the possible instantiationsof $\preceq_{\rho}$. Definition 5.1 is illustrated in Figure 4 .

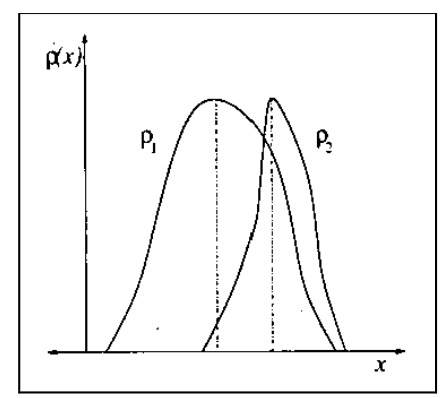

Figure 4: Partial order on set of all fitness distributions: $\rho_{1} \preceq_{\rho} \rho_{2}$ according to Definition $\mathbf{5 . 1}$

Metric $d_{\rho}$ on $\mathrm{Q}$ is defined as follows:

Definition 5.2 Let $\mathcal{Q}$ represent the set of allfiiness distributions. Metric $d,: \mathcal{Q} \times \mathcal{Q} \rightarrow \mathbb{R}$ is defined as

$$
d_{\rho}\left(\rho_{1}, \rho_{2}\right)=\sum_{x \in E_{\rho_{1}} \cup E_{\gamma_{2}}}\left|\rho_{1}(x)-\rho_{2}(x)\right| \quad \forall \rho_{1}, \rho_{2} \in \mathcal{Q}
$$

where $E_{\rho_{1}}$ and $E_{\rho_{2}}$ are finess value sets of $\rho_{1}$ and $\rho_{2}$ respectively.

It is easy to see that $\boldsymbol{d}$, is indeed a metric on $\mathcal{Q}$ since

$$
\begin{array}{r}
\sum_{x \in E_{r_{1}} \cup E_{\rho_{2}}}\left|\rho_{1}(x)-\rho_{2}(x)\right|=0 \Longleftrightarrow \rho_{1}(x)=\rho_{2}(x) \\
\forall \rho_{1}, \rho_{2} \in \mathcal{Q} .
\end{array}
$$

Also the remaining axioms of metric space are easy to verify. Metric $d$, on $\mathcal{Q}$ induces same on $\mathcal{P} / \sim_{p}$ and hence $\left(\mathcal{P} / \sim_{\rho}, d_{\rho}\right)$ is a metric space. We discuss properties of this metric space in $\$ 5.2$.

\subsection{Properties of metric space $\left(\mathcal{P} / \sim_{\rho}, d_{\rho}\right)$}

One property of $d$, is that it takes only nonnegative integer values according to definition of fitness distribution $\rho$. We state this formally as a remark.

\section{Remark 5.3}

$$
\operatorname{range}\left(d_{\rho}\right) \subseteq \mathbb{Z}^{+} \cup\{0]
$$

Also from (8),

$$
\rho^{[P]}=\rho^{P} \quad \forall[P] \in \mathcal{P} / \sim_{\rho},
$$

which means that we can extend the definition of fitness distribution to equivalence classes of populations.

Note that if $P_{1} \sim_{\rho} P_{2}$, then any value of macroscopic parameter of population derived from fitness distribution is equal for $P_{1}$ and $P_{2}$. From (4) all the populations in an equivalence class due to $\sim_{\rho}$ have the same size.

Now we prove a important properly of the $\operatorname{space}\left(\mathcal{P} / \sim_{\rho}\right.$ ,$d_{\rho}$ ), which results due to properties of fitness distribution of population.

Theorem $5.4\left(\mathcal{P} / \sim_{\rho}, d_{\rho}\right)$ is complete.

Proof To prove that $\left(\mathcal{P} / \sim_{\rho}, d_{\rho}\right)$ is complete, we have to prove that every Cauchysequence defined on $\mathcal{P} / \sim_{\rho}$ converges.

Let $\left\{C_{n}\right\}$ be a Cauchy sequence defined on $\mathcal{P} / \sim_{p}$ i.e.,

$$
\begin{gathered}
\forall \epsilon>0, \exists N=N(\epsilon) \in \mathbb{Z}^{+} \ni \\
n, m \geq N \Rightarrow d_{\rho}\left(C_{n}, C_{m}\right)<\epsilon .
\end{gathered}
$$

Choose $\epsilon=1$ i.e.,

$\exists N=N(1) \in \mathbb{Z}^{+} \ni \forall n, m \geq N(1) \Rightarrow d_{\rho}\left(C_{n}, C_{m}\right)<1$.

Since $\boldsymbol{d}$, takes only nonnegative integer values (Remark 5.3), the above statement can be written as,

$$
\exists N=N(1) \in \mathbb{Z}^{+} \ni \forall n, m \geq N(1) \Rightarrow d_{\rho}\left(C_{n}, C_{m}\right)=0
$$

1.e.,

$$
C_{n}=C_{m} \quad \forall n, m \geq N(1)
$$


Thus

$$
\mathrm{n} \geq N(1) \Rightarrow \mathrm{C}^{\prime \prime}=C_{N(1)} \in \mathcal{P} / \sim_{\rho},
$$

which proves the convergence of Cauchy sequence $\left\{C_{n}\right\}$ in $P / \sim_{\rho}$. The claim now follows.

One important property that one would look for in a metric space is 'compactness'. But a metric space is compact if and only if it is complete and totaly bounded [19]. But note that $\left(\mathcal{P} / \sim_{p}, d_{, y}\right)$ need not he a hounded metric space since we have not given any hound on the population size though we simply assumed that it is finite. Unfortunately, even if we give a bound on the population size, $\left(\mathcal{P} / \sim_{\rho}, d_{\rho}\right)$ is hounded hut not totally hounded unless we assume that $\mathcal{P} / \sim_{\rho}$ is finite because $\left(\mathcal{P} / \sim_{\rho}, d_{\rho}\right)$ is a discrete metric space (since $d_{\rho}$ takes only integer values). We state this as a remark.

Remark 5.5 Metric space $\left(\mathcal{P} / \sim_{\rho}, d_{\rho}\right)$ is conipact $\Longleftrightarrow$ $\mathcal{P} / \sim_{\rho}$ is finite.

\subsection{Convergence of monotone evolutionary process}

Now we give a simple result on convergence of monotone evolutionary processes. In the following theorem, we assume that $\Omega$ is finite, which is a valid assumption for genetic algorithms and many other evolutionary algorithms.

Theorem 5.6 Let $\Omega$ be finite and let $\left\{P_{n}\right\}$ be a monotone evolutionay process, then $\left\{P_{n}\right\}$ converges with respect to evolutionay criteria $p$.

Proof: To prove the result we, have to prove that $\left\{\left[P_{n}\right]\right\} \subset \mathcal{P} / \sim_{\rho}$ converges with respect to metric $d_{, .}$. Since $\left\{P_{n}\right\}$ is a monotone evolutionary process, we have the condition that $\left[P_{n}\right] \preceq\left[P_{n+1}\right]$, $\forall n$. First we establish convergence of $\left\{\left[P_{n}\right]\right\}$,

$\left\{\left[P_{n}\right]\right\}$ has finite number of distinct elements. Let that set be $P_{\text {set }}$.

Since $P_{\text {set }}$ is finite (since $\Omega$ is finite) and $\left\{\left[P_{n}\right]\right\}$ is a chain, it has a supremum. Let the supremum be $\left[P_{*}\right] \in \mathcal{P} / \sim_{\rho}$.

$\left[P_{*}\right] \in P_{\text {set }}$, since $P_{\text {set }}$ is finite.

Let $N \in \mathbb{Z}^{+}$he the smallest integer such that $\left[P_{N}\right]=\left[P_{*}\right]$.

Then

$$
n, m \geq N \Rightarrow d_{\rho}\left(\left[P_{n}\right],\left[P_{m}\right]\right)=0
$$

i.e.;

$$
\begin{gathered}
\forall \epsilon>0, \exists N=N(\epsilon) \in Z^{+} \ni \\
n, m \geq N \Rightarrow d_{\rho}\left(\left[P_{n}\right],\left[P_{m}\right]\right)<\epsilon,
\end{gathered}
$$

which implies $\left\{\left[P_{n}\right]\right\}$ is Cauchy.

From Theorem 5.4, $\left\{\left[P_{\mathbf{n}}\right]\right\}$ converges.
Now we prove that $\left[P_{n}\right] \stackrel{d_{\rho}}{\longrightarrow}\left[P_{*}\right]$.

Note that

$$
\mathrm{Ve}>0, n \geq N \Rightarrow d_{\rho}\left(\left[P_{n}\right],\left[P_{*}\right]\right)=0<\epsilon .
$$

The claim follows.

\section{Conclusion}

In this paper we proposed a new mathematical framework to develop theory for evolutionary computation, based on the concept called evolutionary criteria. After formalizing evolutionary criteria we developed the notion of quotient evolutionary space on which we defined evolutionary process. The main characteristic of the proposed mathematical abstraction is to transform the objective that is defined on search space by fitness function to an objective defined on the population space and to measure evolutionary change in terms of macroscopic properties of the population.

The main advantages of the given framework are

- No fixed macro-propertiesof population are assumed; the framework is general enough to consider any macroscopic properties of population that one would be interested in observing.

- Evolutionary process is treated as a strict populational concept, by treating unit of evolutionary change as population.

- By transforming the goal on the search space to populational space, it is easy to understand the evolutionary process.

- Evolutionary process is implicitly treated as an optimization process.

- To achieve the ohjective on the search space, one can design the algorithm in such a way that evolutionary process is reinforced towards the ohjective that is induced on the population space with respect to evolutionary criteria, at each iteration.

To demonstrate the abstraction, we have given a detailed analysis of fitness distrihution as evolutionary criteria and presented the convergence results.

To develop theories for specific evolutionary algorithms, based on the mathematical abstraction that we proposed in this paper, one needs to explore the best possible evolutionary criteria that can he chosen to analyze the specific algorithms. The abstract concepts evolutionay criteria and quotient evolutionary space we proposed in this paper would be useful to develop an unified theory of evolutionary computation. 


\section{Appendix}

\section{A Proof of Theorem 3.2}

Suppose that there is a map $[\mathcal{F}]$ which satisfies (7) then

$$
\begin{array}{r}
{[\mathcal{F}]([P])=j([\mathcal{F}]([P]))=j\left([\mathcal{F}]\left(\pi_{\mathcal{F}}(P)\right)\right)} \\
=\left(j \circ[\mathcal{F}] \circ \pi_{\mathcal{F}}\right)(P) .
\end{array}
$$

From (7), we have

$$
\left(j \circ[\mathcal{F}] \circ \pi_{\mathcal{F}}\right)(P)=\mathcal{F}(P) .
$$

Thus, if it exists, $[\mathcal{F}]$ is uniquely determined and it must be defined by

$$
[\mathcal{F}]([P])=\mathcal{F}(P) \quad \forall P \in P
$$

Now we observe that $\forall P_{1}, P_{2} \in \mathcal{P}$

$$
\left[P_{1}\right]=\left[P_{2}\right] \Leftrightarrow P_{1} \sim_{\mathcal{F}} P_{2} \Leftrightarrow \mathcal{F}\left(P_{1}\right)=\mathcal{F}\left(P_{2}\right) .
$$

Thus the map $|\mathcal{F}|: \mathcal{P} / \sim_{\mathcal{F}} \rightarrow \mathcal{F}(\mathcal{P})$ is well defined by (I 2). Clearly $[\mathcal{F}]$ is on-to; and from $(13),[\mathcal{F}]$ is one-one and hence $[\mathcal{F}]$ is bijective.

\section{Bibliography}

[I] T. E. Davis and J. C.Principe, "A simulated annealing like convergence theory for the simple genetic algorithm," in Proceedings of 4th Conference on Genetic Algorithms, R. K. Belew and L. B. Booker, Eds., San Mateo, CA, 1991, pp. 174-181.

[2] Gunter Rudolph, "Convergence analysis of canonical genetic algorithms," IEEE Transactions on Neural Networks, vol. 5, no. 1, pp. 96-101, January 1994.

[3] Giinter Rudolph, "On a Multi-Objective Evolutionary Algorithm and Its Convergence to the Pareto Set," in Proceedings of the 5th IEEE Conference on Evolutionary Computation, Piscataway, New Jersey, 1998, pp. 511-516, IEEE Press.

[4] Kwong-Sak Leung, Qi-Hong Duan, Zong-Ben Xu, and C. K. Wong, "A new model of simulated evolutionary computation - convergenceanalysis and specifications," IEEE Transactions on Evolutionary Computation, vol. 5, no. 1, pp. 3-16, 2001.

[5] Adam Priigel-Bennett and Jonathan Shapiro. "Analysis of genetic algorithms using statistical mechanics:" Physical Review Letters, vol. 9, no. 72, February 1994.

16) Adam Prugel-Bennett, "Modelling evolving populations," Journal of Theoretical Biology, vol. 185, pp $81-95,1997$
[7] Adam Priigel-Bennett and Alex Rogers, "Modelling ga dynamics," in Theoretical Aspects of Evolutionary Computing, L. Kallel, B. Naudts, and A. Rogers, Eds., pp. 59-86. Springer-Verlag. Berlin Heidelberg. 2001.

[8] Lars Magnus Rattray, "The dynamics of a genetic algorithm under stabilizing selection:' Complex Systems, vol. 9, pp. 213-234, 1995.

[9] P. Moral, L. Kallel, and J. Rowe, "Modeling genetic algorithms with interacting particle systems," in Theoretical Aspects of Evolutionan Computing, L. Kallel, B. Naudts, and A. Rogers, Eds., pp. 10-67. SpringerVerlag, Berlin, 2001.

[10] T. C. Jones, Evolutionan, Algorithms. Fitness Landscapes and Search, Ph.D. thesis, University of New Mexico, Albuquerque, NM, March 1995.

[1 I] Peter F. Stadles and Robert Happel, "Random field models for fitness landscapes," Working Papers 9507-069, Santa Fe Institute, Santa Fe, NM, 1995.

[12] Peter Schuster, "Landscapes and molecular evolution," Physica D, vol. 107, pp. 351-365, 1997.

[13] Wirt Atmar, "Notes on the simulation of evolution," IEEE Transactions on Neural Networks, vol. 5, no. I, pp. 130-148, 1994.

[14] A Keane, "An introduction to evolutionary computation in design, search and optimization," in Theoretical Aspects of Evolutionary Computing, L. Kallel, B. Naudts, and A. Rogers, Eds. Springer-Verlag. Berlin Heidelberg, 2001.

[15] Michael D. Vose, "Modeling simple genetic algorithms," Evolutionan Computation. vol. 3, no. 4, pp. $453-472,1995$.

[16] E. van Nimwegen, J. P. Crutchfield, and M. Mitchel, "Finite populations induce metastability in evolutionary search," Physics Letters A, vol. 229, pp. 144-150, 1997.

[17] Mark A. Bedau and Norman H. Packard, "Measurement of evolutionary activity, teleology, and life," in Artificial Life 2, Santa Fe Institute Studies in the Sciences of Complexity, C. Langton, C. Taylor, D. Farmer, and S. Rasmussen, Eds., vol. 10,pp. 4.31461. Addison-Wesley, Redwood City. CA, 1992.

[18] T. G. Dobzhansky, The Biology of Ultimate Concern, New American Library, 1967.

[19] A. N. Kolmogorov and S. V.Fomin, Introductory Real Analysis. Dover Publications. Mineola. N.Y.. 1975. 